\title{
Diálogo del saber campesino y la investigación científica: árboles nativos dendroenergéticos en la Reserva de la biosfera Sierra de Huautla, Morelos, México
}

Farmer knowledge and scientific research dialogue: dendroenergetic native trees in the Biosphere Reserve Sierra de Huautla, Morelos, Mexico

Dialogue entre savoir paysan et recherche scientifique : arbres indigènes utilisés comme combustibles dans la Réserve de biosphère de la Sierra de Huautla, Morelos, Mexique

Artemio Cruz León, Miguel Uribe Gómez, Alejandro Lara Bueno, César Augusto Yescas Albarrán and Ranferi Maldonado Torres

\section{OpenEdition}

\section{Electronic version}

URL: http://journals.openedition.org/ethnoecologie/2493

DOI: $10.4000 /$ ethnoecologie.2493

ISSN: 2267-2419

Publisher

Laboratoire Eco-anthropologie et Ethnobiologie

\section{Electronic reference}

Artemio Cruz León, Miguel Uribe Gómez, Alejandro Lara Bueno, César Augusto Yescas Albarrán and Ranferi Maldonado Torres, « Diálogo del saber campesino y la investigación científica: árboles nativos dendroenergéticos en la Reserva de la biosfera Sierra de Huautla, Morelos, México », Revue d'ethnoécologie [Online], 9 | 2016, Online since 01 July 2016, connection on 20 April 2019. URL : http:// journals.openedition.org/ethnoecologie/2493; DOI : 10.4000/ethnoecologie.2493

This text was automatically generated on 20 April 2019.

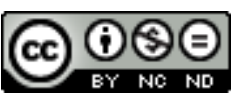

Revue d'ethnoécologie est mis à disposition selon les termes de la licence Creative Commons Attribution - Pas d'Utilisation Commerciale - Pas de Modification 4.0 International. 


\section{Diálogo del saber campesino y la investigación científica: árboles nativos dendroenergéticos en la Reserva de la biosfera Sierra de Huautla, Morelos, México}

Farmer knowledge and scientific research dialogue: dendroenergetic native trees in the Biosphere Reserve Sierra de Huautla, Morelos, Mexico

Dialogue entre savoir paysan et recherche scientifique : arbres indigènes utilisés comme combustibles dans la Réserve de biosphère de la Sierra de Huautla, Morelos, Mexique

Artemio Cruz León, Miguel Uribe Gómez, Alejandro Lara Bueno, César Augusto Yescas Albarrán and Ranferi Maldonado Torres

\section{Introducción}

1 La extracción de especies arbóreas para leña, combustible aún esencial para las familias campesinas de varias regiones de México, constituye un indicador del subdesarrollo rural. En 2007, la extracción de leña en el país ascendió a 38 millones de metros cúbicos, según reporte de la Conafor (Comisión Nacional Forestal 2007). El uso de la leña para los fogones del hogar campesino implica una extracción sostenida del recurso forestal, lo cual genera problemas ecológicos y de sustentabilidad en la preservación de los recursos naturales. A las determinaciones científicas y tecnológicas se unieron los conocimientos tradicionales y empíricos de los habitantes del ejido Los Sauces, en especial de sus amas de casa y leñadores. Resultado de ello es una especie de suma de conocimientos locales y académicos, una conjunción que pudiera definirse como una ecología de saberes. En el desarrollo de este trabajo se entrelazan aspectos científicos y sabiduría popular, otro 
objetivo de los autores fue propiciar una reflexión participativa. El objetivo es establecer las bases de futuras tareas agroforestales destinadas a la reforestación de las especies más vulnerables y a la preservación de la selva baja caducifolia de esta zona de estudio.

La dendroenergía, entendida como energía forestal, es toda la energía obtenida a partir de biocombustibles sólidos, líquidos o gaseosos, primarios o secundarios, derivados de los bosques, plantaciones forestales, arbolados urbanos y árboles en general. Es la energía producida después de la combustión de la madera (leña, carbón, pallets, briquetas, etc.) y corresponde al poder calorífico neto del combustible (Guyat et al. 2004). La extracción y uso de la leña impacta negativamente a los recursos forestales, ya sea para autoconsumo o venta (Ortiz et al. 2012).

3 La calidad de la leña se define como el conjunto de características que permiten diferenciarla como igual, mejor o peor, que las restantes de su especie (Otero et al. 2004). Las dos características principales que debe cumplir para su uso como combustible son: a) humedad vs valor energético, la humedad óptima en la leña debe ser de $20 \%$, se sabe que a mayor humedad menor valor energético; $\mathrm{y} b$ ) densidad vs valor energético, la densidad de la madera de la especie se relaciona con el valor energético, a mayor dureza mayor valor energético, en condiciones óptimas de contenido de humedad de la leña.

4 La mayor parte de los usuarios de leña en México se concentra en los estados de la república donde aún la población rural presenta notorios índices demográficos, como Chiapas, Guanajuato, Guerrero, Hidalgo, Michoacán, Morelos, Oaxaca, Puebla, Quintana Roo, Tabasco, Veracruz y Yucatán (Díaz 2000), con un consumo anual de 38 millones $\mathrm{m}^{3}$ / año (Conafor 2007). La extracción de leña es también una actividad muy acentuada en las comunidades de Morelos. Existen 45 especies empleadas con este fin, que corresponden a 7.5\% del total de las plantas útiles, las de mayor demanda son: tepemezquite o tlahuitol ( Lysiloma divaricatum), palo Brasil (Haematoxylum brasiletto), palo dulce (Eysenhardtia polystachya), y tecolhuixtle (Mimosa benthamii) (Atender 2006).

5 La comunidad de Los Sauces se encuentra dentro de la zona de amortiguamiento de la Reserva de la Biosfera Sierra de Huautla (Rebiosh). Se localiza en el estado de Morelos, entre las latitudes $\mathrm{N} 18^{\circ} 33^{\prime} 08^{\prime \prime}$ y $18^{\circ} 37^{\prime} 00^{\prime \prime}$, y las longitudes W $98^{\circ} 58^{\prime}$ y $98^{\circ} 55^{\prime}$; a una altitud de 1,216 m. Colinda al norte con el ejido Zacapalco, al sur con El Limón de Cuachichinola, al este con Pitzotlan, al noreste con Huitchila y al oeste con El Tepehuaje. Orográficamente, se ubica en el límite del Eje Neovolcánico, por ello además de materiales ígneos, en algunas áreas se encuentran rocas metamórficas y sedimentarias. El suelo predominante es feozem háplico (tierra parda) y la pendiente oscila entre 13 y $23 \%$ en zonas más pronunciadas, en las más planas entre 5 y 8\% (INEGI 2011). 
Figura 1 : TECoLhuIXtLe Mimosa benthamii

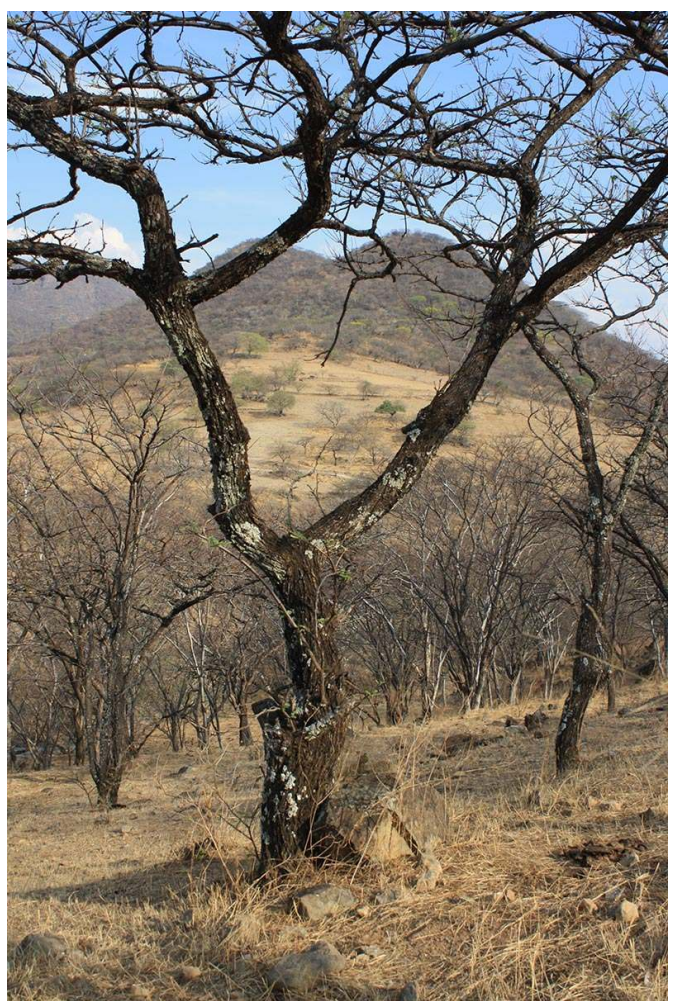

TECOLHUIXTLE, UNA DE LAS ESPECIES DENDROENERgÉTICAS MUY APRECIADA POR LOS LEÑADORES Y LAS AMAS DE CASA; LOS PRIMEROS POR SU CALIDAD Y QUE SE PUEDEN HACER RAJAS Y LAS SEgUNDAS POR EL TIPO DE FLAMA QUE PROPORCIONA Y ALTA CAPACIDAD PARA PRODUCIR CARBÓN.

(C) ARTEMIO CRUZ LEÓN 
Figura 2 : PALO BRASIL Haematoxylum brasiletto

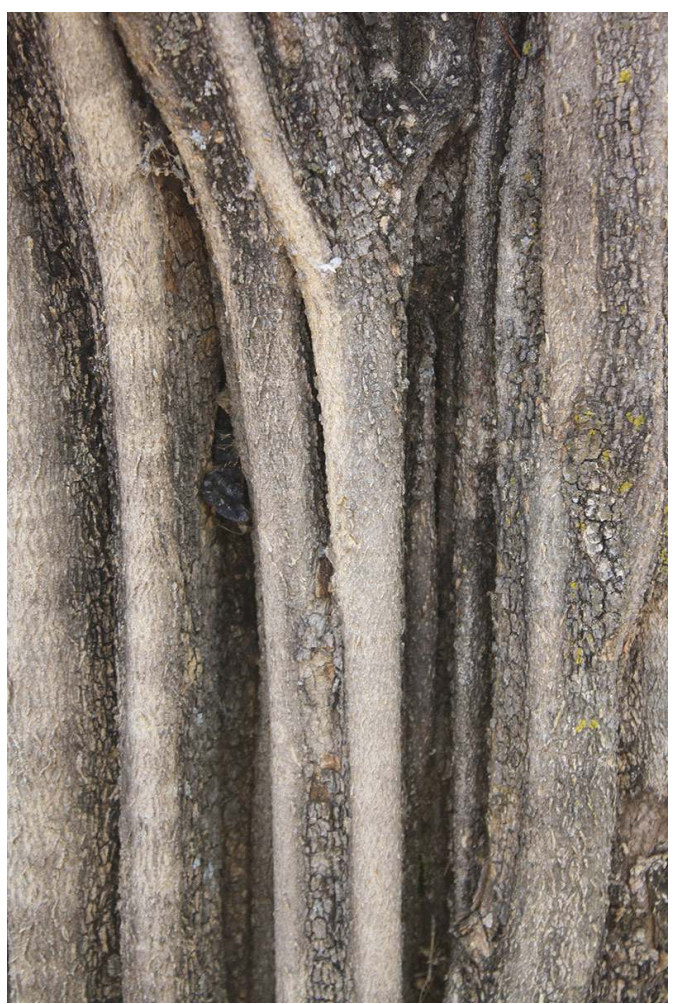

Palo Brasil : ACERCAMIENTO AL TRONCO deL PALO BRASIL, ESPECIE QUE PRODUCE LEÑA ALTAMENTE APRECIADA POR SU CALIDAD CALORífiCA Y SU ALTA CAPACIDAD PARA PRODUCIR RAJAS.

(C) ARTEMIO CRUZ LEÓN

Las principales actividades económicas en el ejido Los Sauces, además de la agricultura y la ganadería, son: recolección de resinas, colecta de frutos y de especies medicinales, producción de mezcal, y extracción de leña. Esta última es utilizada mayormente para autoconsumo, en la cocción de alimentos, y otra parte para su venta entre vecinos o en pueblos aledaños para allegarse ingresos económicos complementarios. La leña es uno de los principales recursos usados como combustible por la mayoría de los habitantes. Es irrelevante anotar que su uso excesivo crea presión sobre la disponibilidad de especies arbóreas y, derivado de un previsible aumento de la población, el pronóstico es que se acelerará su extracción. Esto vulneraría la ya de por sí frágil sustentabilidad de la selva baja caducifolia que existe en la zona y, en especial, la de aquellos ejemplares cuyo destino es la leña. Esta es otra de las razones por la que el objetivo de este trabajo es ubicar árboles nativos con potencial dendroenergético para determinar la calidad de leña en el ejido Los Sauces, municipio de Tepalcingo, Morelos. Además de seleccionar árboles nativos usados por las comunidades por su potencial dendroenergético en este ejido y sistematizar, comparativamente, el conocimiento tradicional de las especies dendroenergéticas y el proceso de aprovechamiento de las mismas que se tiene en dicha comunidad, con los resultados científicos de esta investigación.

7 Propender a que los resultados de este trabajo, eventualmente, sirvan para aminorar la presión extractiva sobre las especies más vulnerables de la selva caducifolia y, paralelamente, como antecedente para cualquier programa posterior destinado a su sustentabilidad, por medio de viveros y reforestación de esas mismas especies arbóreas 
nativas, en acciones que conjunten los saberes e intereses locales con los de los especialistas. Con ese objetivo fueron seleccionadas las especies más apreciadas para su extracción y uso como leña.

\section{Materiales y métodos}

8 Con base en una serie de entrevistas realizadas con anterioridad, durante 2011, se elaboró un cuestionario más detallado para obtener el conocimiento local acerca de las especies más utilizadas como leña (dendroenergéticas). Este cuestionario se aplicó a $25 \%$ de las familias que habitan en la comunidad de Los Sauces. Se efectuaron dos tipos de entrevistas, una para los leñadores y otra para las amas de casa. Esto porque ambos grupos no tienen la misma visión respecto a la leña.

9 Para la obtención de densidad de madera de las especies dendroenergéticas se aplicó la metodología descrita por Kollman (1959); para calor de combustión se utilizó la que reseñan Soto \& Núñez (2008); para cenizas y humedad se usó la descrita por Sosa (1979). El análisis estadístico se realizó mediante el procedimiento GLM de SAS (2002). Las pruebas de comparación de medias con 0.05 de significancia estadística se realizaron mediante la prueba de Tukey (Steel et al. 1997).

\section{Resultados y discusión}

10 Los resultados de las entrevistas realizadas en la comunidad de Los Sauces muestran que existe un proceso parcial de sustitución de la leña por gas butano, que aún no se establece plenamente. También constatan que los intereses y visión acerca de la leña por parte de las amas de casa son diferentes a las de los leñadores. Estos últimos prefieren las especies fáciles de cortar, de rajado lineal y secado rápido, en tanto que las mujeres se inclinan por especies que no producen humo y, en función de sus necesidades, en algunas ocasiones requieren de especies que sean aptas para fuego lento y constante, para cocinar frijoles, $o$ bien leña de fuego rápido, « arrebatado », que se requiere para hacer tortillas.

\section{Importancia de la leña en las comunidades y perspectiva de las amas de casa}

11 Los combustibles son una necesidad tanto en los hogares de comunidades rurales como en los urbanos, que se emplean para la preparación de alimentos, pero también para temperar el agua usada con fines de higiene personal. $Y$ aunque la leña es un combustible más propio y tradicional de las comunidades rurales ésta se va sustituyendo por combustibles fósiles, en la medida que sus habitantes tienen acceso a estos últimos y a los equipos e instalaciones necesarias para su empleo. La leña en las comunidades es un recurso que se maneja de manera apropiada por los habitantes rurales, en la medida que se corta y se procesa para su aprovechamiento, pues los dispositivos para su empleo son muy elementales, elaborados por los propios comuneros. En cambio, el uso de combustibles fósiles requiere equipo e instalaciones que deben ser adquiridos en las ciudades y resultan costosos para los campesinos marginados. Además, la compra de los combustibles requiere pago en efectivo, situación que limita su uso. Por ello, en la localidad de Los Sauces $40 \%$ de los hogares utiliza únicamente leña. No cuentan con 
equipo para combustible fósil, localmente denominado como " gas LP ", que corresponde al butano. Éste se comercializa en cilindros de $20 \mathrm{~kg}$ con un costo actual de 235 pesos. En tanto, $60 \%$ de los hogares combina leña y " gas ", lo cual denota el nivel de transición alcanzado en el cambio de combustibles. A pesar de que la comunidad es productora de leña, incluso para comercializar, ya acusa esa sustitución.

El uso de "gas" para estufas, calentadores de agua y parrillas requiere de espacios cerrados para cocinar y cuartos de baño que se aprecian como parte del prestigio social en la comunidad, éste uso está asociado a la migración de los hijos al extranjero, los recursos de las remesas son destinados para la compra de apararos y construcción especializadas requeridas para el uso de combustible fósil.

Sin embargo, el uso de la leña sigue fuertemente arraigado a la cultura doméstica y al "gusto" de las amas de casa. La encuentran irreemplazable para cocinar, ya que la asocian al « mejor sabor » que logran los alimentos o bien a la obtención de fuego lento o " arrebatado ", según el caso, necesario para la cocción de determinados platillos. Por ello, $90 \%$ de las amas de casa prefieren la leña para cocinar, identifican la leña de especies botánicas de acuerdo a las necesidades de uso y deciden en qué circunstancias usar gas.

La cantidad de leña utilizada depende del número de miembros de la familia: las pequeñas usan sólo de una a dos « cargas » al mes (Una carga son 40 leños de $1 \mathrm{~m}$ de largo, pesa 80 $\mathrm{kg}$ y su volumen es de $0.17 \mathrm{~m}^{3}$ ), en tanto que las extendidas pueden ocupar seis. Resalta el hecho de que $90 \%$ de los entrevistados emplea de una a dos cargas al mes.

La eficiencia en el uso de la leña es una preocupación de los programas gubernamentales, cuya aplicación -junto a la masificación de orientaciones medioambientales- ha dado como resultado que alrededor de $50 \%$ de las familias de esta comunidad cuenten con fogones ahorradores de leña, los cuales consumen sólo la mitad de los tradicionales.

Las especies dendroenergéticas son reconocidas por las amas de casa por su intensidad de fuego: las de fuego «lento », constante y duradero, hacen brasas y no producen humo. Se emplean en la de cocción de frijoles (Cuadro 1).

Cuadro 1 : Especies dendroenergéticas: tipo de fuego, frecuencia y tiempo de secado

\begin{tabular}{|l|c|c|c|c|}
\hline Nombre común & Nombre cientifico & Caracteristicas de la leña & Frecuencia (\%) & Tiempo de secado \\
\hline Tlahuitol & Lysiloma divaricatum & Arde lento, no hace humo & 100 & 15 dias \\
\hline Palo Brasil & Haematoxylum brasiletto & Arde lento & 90 & 3 dias \\
\hline \multirow{2}{*}{ Tepehuaje } & Lysiloma acapulcense & Arde lento, hace brasas & 90 & 15 dias \\
\hline Palo dulce & Eysenhardtia polystachya & Arde lento, hace brasas & 60 & 4 dias \\
\hline Tecolhuixtle & Mimosa benthamii & Hace brasas, no hace humo & 50 & 8 dias \\
\hline Cuahulote & Guazuma ulmifolia & No hace humo & 20 & 8 dias \\
\hline
\end{tabular}

Fuente: Elaboración propia con información de campo

17 En contraste, la leña que arde rápido es preferida para la preparación de alimentos que no requieren mucho tiempo en el fogón, como las tortillas o el recalentado de comida. La leña de cubata blanca reúne estas características, además del cuahulote y el tlahuitol. Hay preferencia por especies que no hacen humo, a pesar de que por naturaleza la leña lo produce, por ello se eligen aquellas cuya emanación es mínima; el secado previo de la leña pretende incrementar la capacidad calórica y reducir la emisión de humo. 


\section{Perspectiva de los leñadores}

18 En la región, la responsabilidad de recolectar leña es una función de género que corresponde a los hombres, pero ocasionalmente algunas mujeres la realizan. Por ello, los valores y significados relacionados con la obtención de leña corresponden a la visión de los hombres, misma que difiere de la que manifiestan las mujeres y que ha sido descrita parcialmente. La valoración de los leñadores toma en cuenta distintas características, como se observa en el cuadro 2. Estas son, entre otras: la demanda de los compradores, la facilidad de corte y rajado, el rendimiento, la obtención de trozos derechos, el mínimo tiempo de secado y la abundancia o tamaño; todas ellas permiten disminuir el esfuerzo de corte y con ello el tiempo empleado. Así, las especies de mayor demanda por los leñadores son: tlahuitol, palo Brasil y tepehuaje, que por tener mayor tamaño rinden más cantidad de leña por árbol. La cubata blanca se prefiere por su facilidad de corte, es muy «blandita» y posee ramas y tallos derechos, según don Margarito Tajonar Pliego, comunero y leñador de Los Sauces.

Cuadro 2 : Especies dendroenergéticas: comportamiento y frecuencia de uso

\begin{tabular}{|l|c|c|c|}
\hline Nombre común & Nombre científico & Características & Frecuencia (\%) \\
\hline Cubata blanca & Acacia pennatula & Fácil de cortar, árbol derecho & 100 \\
\hline Tepehuaje & Lysiloma acapulcense & Demanda para venta, árbol grande & 90 \\
\hline Tlahuitol & Lysiloma divaricatum & Abundancia en rajas & 90 \\
\hline Palo dulce & Eysenhardtia polystachya & Tarda poco en secarse & 80 \\
\hline Tecolhuixtle & Mimosa benthamii & Buen sabor a los alimentos & 60 \\
\hline Palo Brasil & Haematoxylum brasiletto & Rápido en secarse & 50 \\
\hline Cuahulote & Guazuma ulmifolia & Abundancia de la especie & 20 \\
\hline Encino & Quercus sp. & Difícil de cortar y escaso & 20 \\
\hline
\end{tabular}

Fuente: Elaboración propia con información de campo leña; sin embargo, en la medida que los árboles duros son de más tamaño, permiten obtener más leña. El tiempo usado para cortar una carga de leña oscila entre una y dos horas; la mayoría de leñadores corta de una a dos cargas por día y los árboles a cortar deben tener entre cinco y 10 años de edad, con lo cual se garantiza rendimiento y calidad de la leña.

Por tanto, existen diferencias en la preferencia de especies para leña entre amas de casa y leñadores, mismas que se basan en la perspectiva de interés de cada uno de ellos y en la forma como establecen las relacionarse con el combustible.

Las especies que se usan para leña tienen además usos como forraje, madera, medicinal, cerco vivo y sombra, como se aprecia en el cuadro 3. 
Cuadro 3 : Otros usos de las especies dendroenergéticas en el ejido Los Sauces

\begin{tabular}{|l|c|c|c|c|c|c|}
\hline Nombre común & Forrajero & Cerca viva & Postes & Medicinal & Sombra & Maderable \\
\hline Palo Brasil & & $\mathrm{X}$ & & $\mathrm{X}$ & \\
\hline Tepehuaje & $\mathrm{X}$ & $\mathrm{X}$ & $\mathrm{X}$ & & $\mathrm{X}$ & \\
\hline Cubata blanca & $\mathrm{X}$ & $\mathrm{X}$ & & & $\mathrm{X}$ & \\
\hline Palo dulce & $\mathrm{X}$ & $\mathrm{X}$ & $\mathrm{X}$ & $\mathrm{X}$ & & $\mathrm{X}$ \\
\hline Tecolhuixtle & & $\mathrm{X}$ & $\mathrm{X}$ & & $\mathrm{X}$ & \\
\hline \begin{tabular}{l} 
Cuahulote \\
\cline { 2 - 7 }
\end{tabular} & $\mathrm{X}$ & $\mathrm{X}$ & & $\mathrm{X}$ & $\mathrm{X}$ & \\
\hline Encinuitol & $\mathrm{X}$ & & & & & \\
\hline
\end{tabular}

Fuente: Elaboración propia con información de campo

Los resultados de este trabajo coinciden con los de Maldonado (1997) en relación al orden de preferencia de especies que se utilizan como leña en la REBIOSH, las cuales son tlahuitol, tepehuaje, tecolhuixtle, palo Brasil y palo dulce, y en el criterio de selección por baja producción de humo.

\section{Criterios de calidad de las especies dendroenergéticas}

23 Al evaluar las especies dendroenergéticas de forma cuantitativa, con los criterios de calor de combustión, cenizas, humedad y densidad, analizadas estadísticamente con una prueba de Tukey $(\mathrm{p} \leq 0.05)$, se obtiene el cuadro 4 .

Cuadro 4 : Especies dendroenergéticas: composición química, calor de combustión y densidad de la madera

\begin{tabular}{|l|c|c|c|c|c|}
\hline Especie & ${ }^{*}$ Materia seca \% & ${ }^{*}$ Materia orgánica \% & ${ }^{*}$ Cenizas \% & ${ }^{*}$ Calor de combustión (cal/g) & ${ }^{*}$ Densidad g/100 $\mathbf{c m}^{\mathbf{3}}$ \\
\hline Cubata blanca & 89,95 & 82,53 & 7,47 & 3894,5 & 0,66 \\
\hline Palo Brasil & 92,06 & 86,61 & 3,39 & 4182,5 & 0,81 \\
\hline Tepehuaje & 93,65 & 87,69 & 2,31 & 4429,0 & 0,58 \\
\hline Tlahuitol & 93,04 & 88,14 & 1,86 & 4215,5 & 0,373 \\
\hline Tecolhuixtle & 0,65 & 86,60 & 3,40 & 4490,0 & \\
\hline
\end{tabular}

* Los valores de atributos estudiados para cada especie marcados con letras diferentes son significativamente diferentes, según la prueba de Tukey $(a=0,05)$. Los promedios se muestran con el límite de confianza al $95 \%$

Fuente: Elaboración propia con información de campo

\section{Calor de combustión de las especies dendroenergéticas}

El calor de combustión es la liberación de energía en forma de calor, que tiene como consecuencia que los alimentos se cocinen. El análisis estadístico arrojó que al menos un tratamiento es diferente. Aplicando la prueba de Tukey $(\mathrm{p} \leq 0.05)$ se obtienen tres grupos en el listado de las especies dendroenergéticas, donde tecolhuixtle y tepehuaje poseen el calor de combustión mayor (4,490 y 4,429 cal.g ${ }^{-1}$ respectivamente). Drake et al. (2002) mencionan que la leña libre de agua posee un poder calorífico inferior de hasta 4,400 cal.g ${ }^{-1}$. En los datos de laboratorio que se obtuvieron, las especies antes mencionadas presentan un valor por arriba del que señala el autor y las siguientes cuatro especies están por debajo.

En los resultados sobre las preferencias en el uso de las especies para leña, contrastados con los datos de laboratorio (Figura 3), corresponde al tepehuaje el segundo lugar en cantidad de calor de combustión y segundo lugar en la preferencia de uso entre las 
familias del ejido de Los Sauces. Esta coincidencia entre las dos formas de obtener el conocimiento de las características de las especies para leña habla de la posibilidad de establecer un diálogo de saberes.

Figura 3 : Calor de combustión de las especies dendroenergéticas

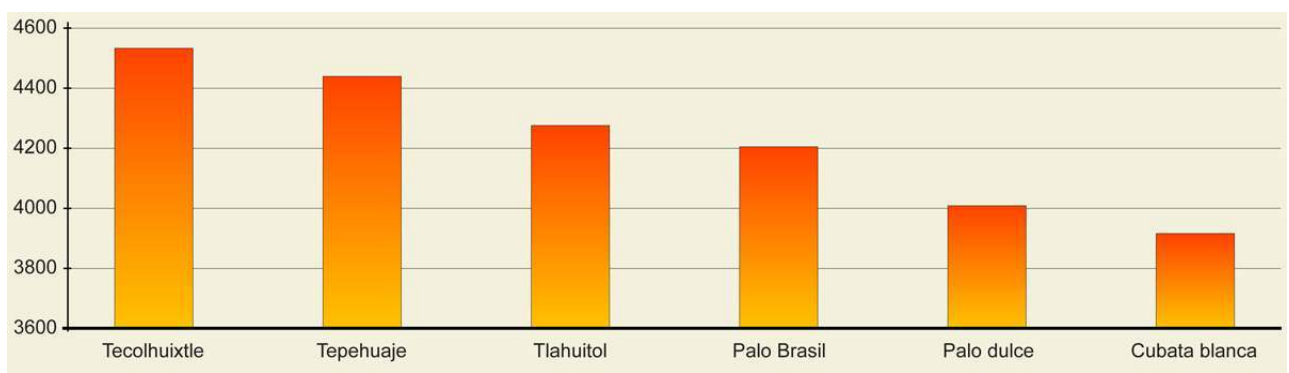

Fuente: Elaboración propia con información de campo

\section{Cantidad de cenizas y materia orgánica}

Sosa (1979) establece que a menor cantidad de cenizas mayor cantidad de materia orgánica y mayor calor de combustión, siendo la materia orgánica responsable de la cantidad de calor de combustión (Bravo 1989). Al comparar los datos obtenidos de calor de combustión y cenizas hay coincidencia en este principio para tepehuaje y tlahuitol (Figura 4). El análisis estadístico de los resultados, revela que al menos un tratamiento es diferente, al aplicar la prueba de Tukey $(\mathrm{p} \leq 0.05)$ se obtiene que existen cuatro grupos, donde nuevamente tepehuaje y tlahuitol, además de contener la menor cantidad de cenizas, poseen la mayor cantidad de materia orgánica, por lo cual sus características de producción de calor son mejores, de allí su preferencia. Nuevamente, la preferencia de estas dos especies como leña, por su baja emisión de humo y calidad en calor, se deriva de un conocimiento campesino de los usuarios que, al contrastarlo con conocimiento científico, encuentra coincidencia; la preferencia de las amas de casa se basa en la menor producción de cenizas y humo, y la mayor cantidad de metería orgánica que significa mejor calor. El humo contiene más de 200 compuestos químicos, la mayoría de ellos tóxicos e inhalables con un diámetro menor a 10 micrones, causa de problemas respiratorios (Junemann \& Legarreta 2007).

Figura 4 : Relación entre la cantidad de cenizas y materia orgánica

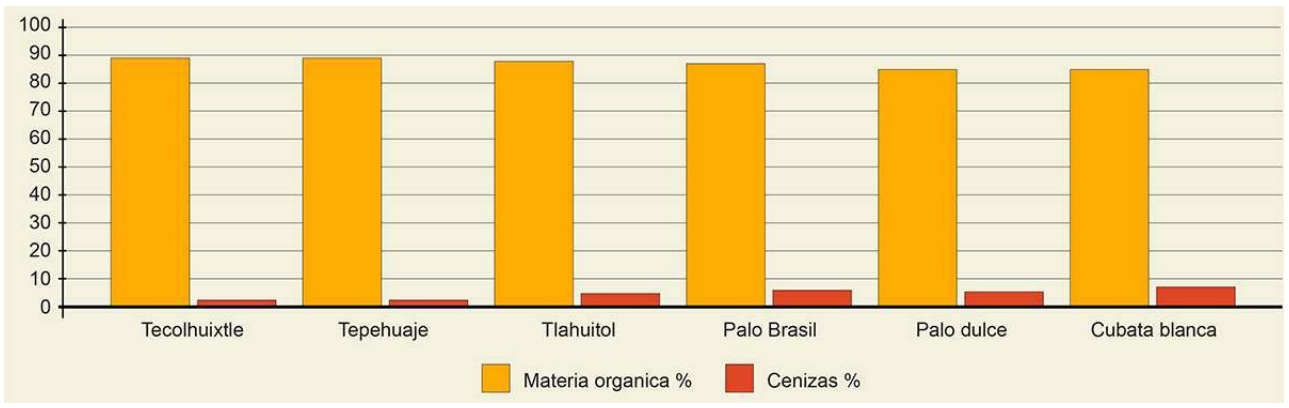

Fuente: Elaboración propia con información de campo

Restrepo et al. (1983) mencionan que el análisis de la composición del humo de leña ha demostrado que se trata de una suspensión de partículas pequeñas en aire caliente y otros 
gases, resultado de una combustión incompleta. Los gases son variables pero siempre contienen monóxido de carbono y dióxido de carbono; si hay azufre, así sea en pequeñas cantidades, se produce dióxido de azufre, vapores de alquitrán y/o hidrocarburos insaturados. Las partículas de carbón están recubiertas de materiales combustibles tales como ácidos orgánicos y aldehídos. Un caso de problemas respiratorios a causa de la inhalación excesiva de humo se aborda en la investigación de Chacón \& Alfaro (1992), es un estudio con mujeres (de alrededor de 30 años) internadas en la clínica Guanacaste, Costa Rica. Éstas padecían una afección respiratoria llamada neumopatía, causada por la inhalación excesiva y continuada de humo en los fogones.

\section{Contenido de humedad de las especies dendroenergéticas}

De acuerdo a contenido de humedad, todas las especies tienen valores por abajo del $20 \%$ establecido por Otero et al. (2004) y Escobar et al. (2009). Se sabe que con humedad menor a $20 \%$ se obtiene mayor calor de combustión. En cuanto a la diferencia de tratamientos, analizando estadísticamente los resultados obtenemos que al menos un tratamiento es diferente. Aplicando la prueba de Tukey $(\mathrm{p} \leq 0.05)$ observamos en la figura 5 que hay cinco grupos, donde Tepehuaje y Tlahuitol tienen el menor contenido de humedad (6.36 y $6.96 \%$, respectivamente). Estas dos especies poseen las características necesarias para la producción de mayor calor de combustión, este dato explica la preferencia de las amas de casa.

Figura 5 : Contenido de humedad de especies dendroenergéticas

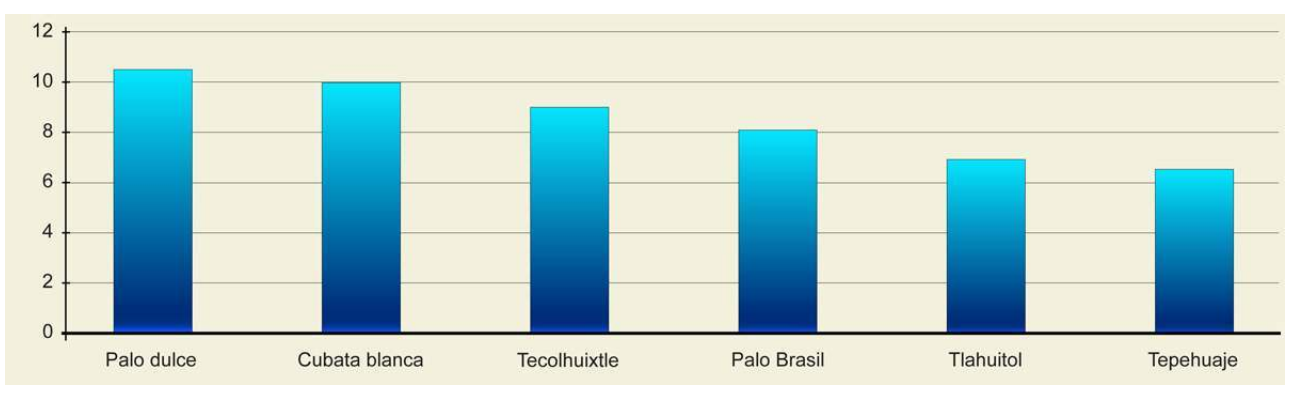

Fuente: Elaboración propia con información de campo

\section{Densidad de las especies dendroenergéticas}

El peso de la unidad de volumen de la madera se asocia a la densidad (Bruzos 2009). Otero et al. (2004) mencionan que a mayor densidad mayor calor de combustión, sin embargo en la Sierra de Huautla las especies con mayor calor de combustión no corresponden a las de mayor densidad. De acuerdo a los datos de la figura 6, palo Brasil y palo dulce son las de mayor densidad ( 0.81 y $0.78 \%$, respectivamente), sin embargo no tienen mayor calor de combustión. De acuerdo con Zhang (1997), la densidad se relaciona con la dureza y calidad de la madera. Este dato coincide con el conocimiento local, ya que el orden de las especies por su dureza, de mayor a menor, son: palo Brasil, tlahuitol, palo dulce, tecolhuixtle, tepehuaje y cubata blanca. 
Figura 6 : Densidad de especies dendroenergéticas

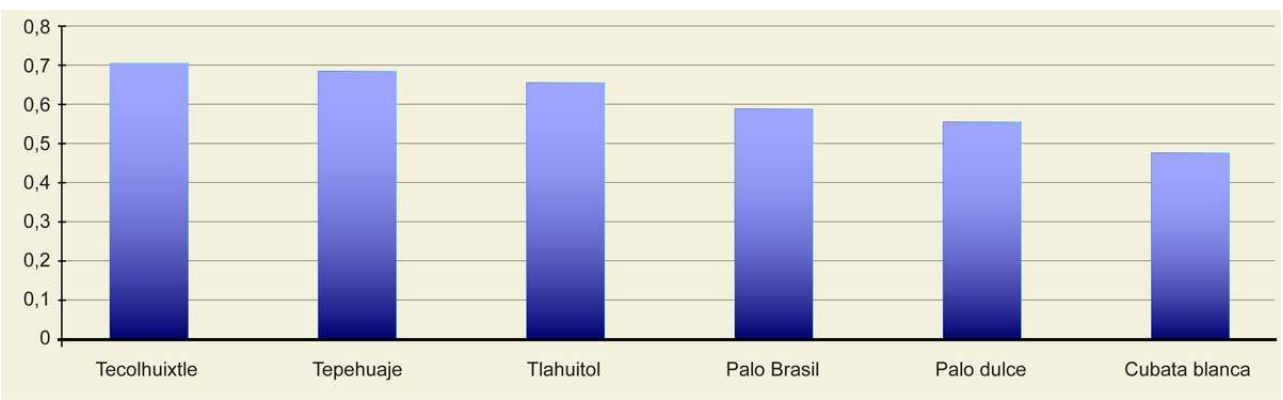

Fuente: Elaboración propia con información de campo

\section{Conclusiones}

cubata blanca, tepehuaje, palo Brasil, palo dulce, y tecolhuixtle. Existe un proceso gradual de sustitución de leña por combustible fósil; sin embargo, a pesar del uso cada vez más extendido de éste, se utiliza esporádicamente. Se percibe como parte del « prestigio social ». También existen diferencias en cuanto a preferencia de especies entre amas de casa y leñadores. Con base en los resultados del análisis de calidad de la leña, las especies de mayor a menor son: tepehuaje, tlahuitol, tecolhuixtle, palo Brasil, palo dulce y cubata blanca.

El uso de especies dendroenergéticas no sólo depende del calor de combustión, cenizas, humedad y densidad sino de otros factores culturales, como la preferencia por la cubata blanca y el tecolhuixtle. Aunque no las califican como de muy buena calidad, las amas de casa mencionan que al cocinar con leña de tales especies los alimentos adquieren un sabor diferente y mejor.

Señalar las especies preferidas para leña también tuvo como finalidad indicar que son ellas el objeto de cualquier programa agroforestal, en especial aquellas más vulnerables, como el tecolhuixtle, cuya población ha disminuido notoriamente, o la cubata blanca, por ser preferida tanto por las amas de casa como por los leñadores.

\section{BIBLIOGRAPHY}

Atender (Asistencia Técnica para el Desarrollo del Campo A. C.) 2006 - Aprovechamiento doméstico y comercial de leña y postería en la selva baja caducifolia del estado de Morelos. Temixco, Morelos. 513 p. [En ligne] http://sinat.semarnat.gob.mx/dgiraDocs/documentos/mor/estudios/2006

Bravo G. L. R. 1989 - Proceso de degradación térmica de la madera: revisión y potencial futuro. In : Primera Reunión Nacional sobre Dendroenergía. Resúmenes de ponencias. Chapingo, México, Universidad Autónoma Chapingo : 348-362.

Revue d'ethnoécologie, 9 | 2016 
Bruzos T. 2009 - Propiedades físicas de la madera. Maderas: Ciencia y Tecnología 11 (1) : 3-18.

Chacón C. R. \& Alfaro R. J. C. 1992 - Neumopatía asociada a la inhalación de humo de leña: Análisis de 11 casos. Revista Costarricense de Ciencias Médicas 13 (3/4) : 7-13. [En ligne] http:// www.binasss.sa.cr/revistas/rccm/v13n3-4/art2

Conafor 2007 - Programa Nacional de Dendroenergía Forestal 2007-2012. 12 p. [En ligne] http:// www.cleancookstoves.org/resources_files/programa-nacional-de

Díaz R. 2000 - Consumo de leña en el sector residencial de México. Evolución histórica y emisiones de $\mathrm{CO}_{2}$. Tesis Maestría en Ingeniería (energética), División de Estudios de Posgrado, Facultad de Ingeniería, UNAM, México, D. F. 113 p.

Drake F., Von B. D., Hellwig M. \& Mellado A. 2002 - Cadena de consumo de leña. Colombia, Departamento de Análisis Instrumental, Universidad de Concepción, 109 p. [En ligne] http:// www.sinia.cl/1292/articles-28474_recurso_1

Escobar O. M. C., Niños C. J. A., Ramírez M. N. \& Yépez P. C. 2009 - Diagnóstico participativo del uso y abastecimiento de leña en Chiapas, México. Revista Autónoma Indígena de México 5 (2) : 201-223. [En ligne] http://revistas.unam.mx/index.php/rxm/article/view/7015/6523

Guyat M. A., Mercadet A. \& Padrón R. 2004 - La dendroenergía: consideraciones generales. Revista Forestal Baracoa 23 : 129-136. [En ligne] http://agris.fao.org/agris-search/search.do? recordID=CU2010800111

INEGI 2011 - Anuario estadístico Morelos 2005. México. [En ligne] http://www.inegi.org.mx/est/c ontenidos/espanol/sistemas/aee10/estatal/mor/defult.htm

[Consultado octubre 2013]

Junemann A. y Legarreta G. 2007 - Inhalación de humo de leña: una causa relevante pero poco reconocida de enfermedad pulmonar obstructiva crónica. División Neumonología, Hospital de Clínicas, Universidad de Buenos Aires 2 : 51-57. [En ligne] http://www.ramr.org.ar/archivos/ numero/ano_7_2_dic_2007/

Kollman F. 1959 - Tecnología de la madera y sus aplicaciones. Tomo I. Ministerio de Agricultura. Madrid, España, Instituto Forestal de Investigaciones y Experiencias y Servicios de la Madera : 359-447.

Maldonado B. J. 1997 - Aprovechamiento de los recursos florísticos de la Sierra de Huautla, Morelos, México. Tesis de Maestría. UNAM, México. 140 p.

Ortiz P. R., Fuentes F. N. A. \& Leyva C. O. 2012 - Extracción y uso de la leña en Yucatán, México: un enfoque de simulación dinámica. Quantitativa Revista de Economía 2 (1) : 39-65. [En ligne] http://quantitativa.ucol.mx/index.php/Quantitativa/issue/view/5

Otero D. L., Lobos B. M., Vera S. A. \& Kausel K. T. 2004 - Estudio generación de antecedentes para la implementación de un sistema nacional de certificación de leña. Temuco, Chile, CONAMA Región de La Araucanía, 91 p. [En ligne] http://www.sinia.cl/1292/articles-46038_recurso_1

Restrepo. J., Reyes. P., Ochoa P. de \& Patiño E. 1983 - Neumoconiosis por inhalación de humo de leña. Acta Médica 8 : 191-204.

SAS 2002 - SAS User's Guide: Statistics (Release 8.02). Cary, NC, USA, SAS Inst., Inc.

Sosa M. E. 1979 - Manual de procedimientos analíticos para alimentos de consumo animal. Chapingo, México, Universidad Autónoma Chapingo, 115 p.

Soto G. \& Núñez M. 2008 - Fabricación de pellets de carbonilla, usando aserrín de Pinus radiata. Maderas: Ciencia y Tecnología. 10 (2) : 129-137. 
Steel G.D.R., Torrie J.H. \& Dickey D.A. 1997 - Principles and procedures of statistics: A biometrical approach. The McGraw-Hill Co. Inc, $637 \mathrm{p}$.

Zhang S. Y. 1997 - Wood Quality: Its definition, impact and implications for value. Added timber management and end uses. In : S Y Zhang R Grosselin and G Chauret Timber management towar wood quality and product value Procedings of the CTIA/IUFRO International Wood Quality Workshop. Quebec City. Part I : 17- 39. [En ligne] http://books.google.com.mx/books/about/

Timber_Management_Toward_Wood_Quality_an.html?id=XFZAHQAACAAJ\&redir_esc=y

\section{ABSTRACTS}

The farming communities of the Sierra de Huautla, keep tradition in using firewood, which is strong pressure on the native deciduous trees. Through participatory dialogue with housewives and loggers and their local knowledge, 6 tree species were selected with value fuelwood in order to design agroforestry technologies that promote the use and the preservation. The species are: tlahuitol [Lysiloma divaricatum (Jacq.) Willd.], tepehuaje [Lysiloma acapulcense (Kunth) Benth.], palo Brasil (Haematoxylum brasiletto H.Karst.), palo dulce [Eysenhardtia polystachya (Ortega) Sarg.], tecolhuixtle (Mimosa benthamii J.F.Macbr.) and cubata blanca [Acacia pennatula (Schltdl. \& Cham.) Benth.]. The contents of dry matter, moisture, organic matter, ash, as well as the heat of combustion and wood density rates, indicators of the quality of the wood, were measured for these species. Tecolhuixtle is the most diminished species by its highly desirable characteristics as firewood; currently, cubata blanca presents intense extraction, although the best species of wood for housewives and loggers are tlahuitol and tepehuaje.

Las comunidades campesinas de la Sierra de Huautla mantienen la tradición del uso de leña, lo cual ejerce fuerte presión sobre los árboles nativos de la selva baja caducifolia. Mediante el diálogo participativo con amas de casa y leñadores, y a partir de sus saberes locales, se seleccionaron seis especies arbóreas con valor dendroenergético para el diseño de tecnologías agroforestales que promovieran el uso y la conservación. Las arbóreas son: tlahuitol [Lysiloma divaricatum (Jacq.) Willd.], tepehuaje [Lysiloma acapulcense (Kunth) Benth.], palo Brasil ( Haematoxylum brasiletto H.Karst.), palo dulce [ Eysenhardtia polystachya (Ortega) Sarg.], tecolhuixtle (Mimosa benthamii J.F.Macbr.) y cubata blanca [Acacia pennatula (Schltdl. \& Cham.) Benth.]. De estas especies se determinaron los contenidos de materia seca, humedad, materia orgánica, cenizas, así como los índices de calor de combustión y densidad de la madera, indicadores de la calidad de la leña. Tecolhuixtle es la especie disminuida por sus características altamente deseables como leña, pero actualmente cubata blanca presenta intensa extracción, aunque las mejores especies dendroenergéticas para las amas de casa y leñadores fueron tlahuitol y tepehuaje.

Les communautés paysannes de la Sierra de Huautla maintiennent la tradition de l'utilisation du bois de chauffage, ce qui exerce une forte pression sur les espèces indigènes de la forêt caducifoliée. Grâce à un dialogue participatif avec les ménagères et les bûcherons, et à partir de leur connaissance locale, six espèces d'arbres combustibles ont été sélectionnées pour concevoir des technologies agroforestières qui favorisent l'utilisation et la conservation. Les espèces sont tlahuitol [Lysiloma divaricatum (Jacq.) Willd.] tepehuaje [Lysiloma acapulcense (Kunth) Benth.] pernambouc (Haematoxylum brasiletto H.Karst, palo dulce [Eysenhardtia polystachya (Ortega) Sarg.] tecolhuixtle (Mimosa benthamii J.F.Macbr.) et cubata blanca [Pennatula Acacia (Schltdl. \& Cham.) Benth.]. Pour ces espèces ont été déterminées la teneur en matière sèche, l'humidité, la matière organique, les cendres, également les indices de chaleur de combustion et de densité du bois qui sont des indicateurs de qualité du combustible. Tecolhuixtle est l'espèce qui a le plus 
diminué à cause de ses caractéristiques hautement prisées comme combustible, mais actuellement cubata blanca fait l'objet d'une exploitation intense, bien que les meilleures espèces dendroénergétiques pour les ménagères et les bûcherons soient tlahuitol et tepehuaje.

\section{INDEX}

Geographical index: Sierra de Huautla, mexique

Mots-clés: dialogue des savoirs, qualité du bois, espèces indigènes, agroforesterie, Mexique Palabras claves: diálogo de saberes, calidad de leña, especies nativas, agroforestería, México Keywords: dialogue of knowledges, quality wood, native species, agroforestry, Mexico

\section{AUTHORS}

\section{ARTEMIO CRUZ LEÓN}

Universidad Autónoma Chapingo, km 38.5 Carretera México-Texcoco, Chapingo, Estado de México.C.P.56230. etnoagronomia1@gmail.com

MIGUEL URIBE GÓMEZ

Universidad Autónoma Chapingo

ALEJANDRO LARA BUENO

Universidad Autónoma Chapingo

\section{CÉSAR AUGUSTO YESCAS ALBARRÁN}

Universidad Autónoma Chapingo

\section{RANFERI MALDONADO TORRES}

Universidad Autónoma Chapingo 